\title{
Nasopharyngeal carcinoma in a six-year-old female child: a case report
}

\author{
Santosh Kumar Swain', Smrutipragnya Samal2, Mahesh Chandra Sahu² \\ 'Department of Otorhinolaryngology, IMS and SUM Hospital, Siksha "O" Anusandhan University, K8, Kalinganagar, \\ Bhubaneswar, Odisha, India \\ ${ }^{2}$ Medical Research Laboratory, IMS and SUM Hospital, Siksha "O" Anusandhan University, K8, Kalinganagar, Bhubaneswar, \\ Odisha, India
}

\section{ABSTRACT}

Nasopharyngeal carcinoma (NPC) is an extremely rare malignancy among the paediatric age group. The relative rarity of paediatric NPC makes the diagnosis difficult. Consequently, NPC tends to be locally advanced at the time of diagnosis in the paediatric age group and is sometimes associated with distant metastasis. The diagnosis of paediatric NPC should prompt immediate treatment. We report a case of undifferentiated nasopharyngeal carcinoma in a young girl who presented with advanced loco-regional disease and was treated with chemoradiation.

\section{KEY WORDS:}

paediatric age, nasopharyngeal carcinoma, chemoradiation.

\section{INTRODUCTION}

Nasopharyngeal carcinoma (NPC) is a malignant neoplasm arising from the epithelial cells lining the nasopharynx. It is a rare malignancy with incidence in highrisk areas at 20 per 100,000 persons per year and lower rates seen in paediatric age [1]. NPC is a rare tumour in children younger than 10 years of age, and the incidence rises gradually with the age of the patient. It is a common head and neck malignant tumour seen in adults, and its incidence varies in different parts of the world. The highrisk areas for nasopharyngeal carcinoma are Southern China, Taiwan, and Hong Kong. NPC is extremely rare among children regardless of the region of the world. Approximately $5 \%$ of primary malignant neoplasms in children arise from the head and neck area [2], whereas NPC represents around $2 \%$ of the head and neck malignant neoplasms in the paediatric age group [1]. Because this is rare in the paediatric age group and because of its non-specific clinical presentations, like nasal congestion, lymphadenopathy, and ear symptoms, the diagnosis is often delayed because of the initial presumption of benign aetiologies. The standard treatment of NPC is radiotherapy. Here we are reporting a case of NPC in a six-year-old girl presenting with nasal block, nasal bleeding, and neck node metastasis.

\section{CASE REPORT}

A six-year-old female child was brought to the outpatient Department of Otorhinolaryngology with complaints of bleeding from the nose, nasal block, and mouth breathing for the preceding four months. The child also presented with right side neck swelling for the preceding two months (Fig. 1). The child had been treated with antibiotics, antihistamines, and nasal decongestants by

\section{ADDRESS FOR CORRESPONDENCE:}

Santosh Kumar Swain, Department of Otorhinolaryngology, IMS and SUM Hospital, Siksha "O" Anusandhan University, K8, Kalinganagar, 751003 Bhubaneswar, Odisha, India, ORCID: 0000-0001-7933-4414, e-mail: santoshvoltaire@yahoo.co.in 


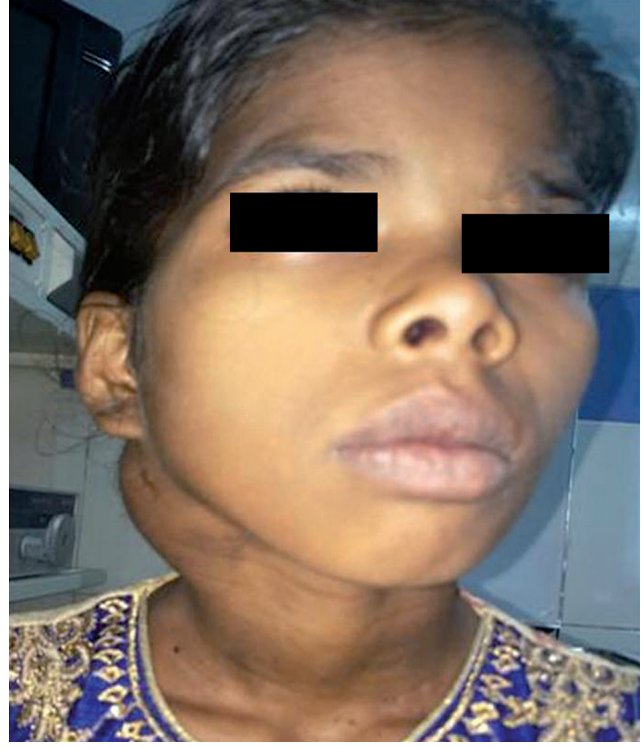

FIGURE 1. A six-year-old girl with nasopharyngeal carcinoma presenting with right-side neck mass

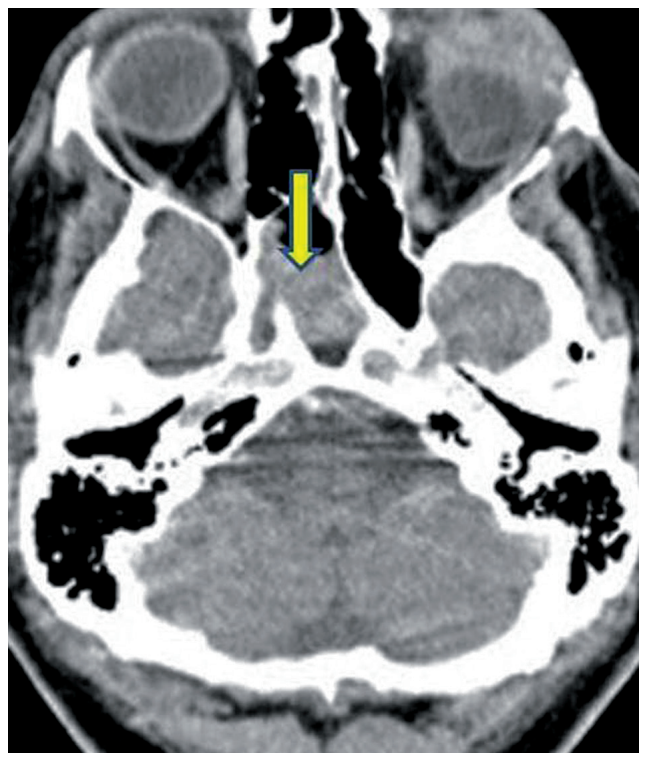

FIGURE 3. (T scan of the nasopharynx and neck showing a mass at the nasopharynx (arrow mark)

local physicians for the preceding four months, but the treatment was unsuccessful. Examination of the oral cavity and oropharynx were within normal limits. On examination of the neck, a $4 \mathrm{~cm} \times 3 \mathrm{~cm}$ hard and fixed mass was seen on the right jugulodigastric area. Fibreoptic nasopharyngolaryngoscopy showed a friable mass at the right side of the nasopharynx, blocking the entry to the oropharynx (Fig. 2). Subsequent CT scan of the head and neck region revealed a large nasopharyngeal tumour approximately $4 \mathrm{~cm}$ in size with locally advanced extension and invasion (Fig. 3). The right side jugulodigastric node was enlarged to around $3 \mathrm{~cm}$. The child was posted for nasopharyngeal biopsy under general anaesthesia and fine needle aspiration cytology from the enlarged neck node. The histopathology report showed invasive cords

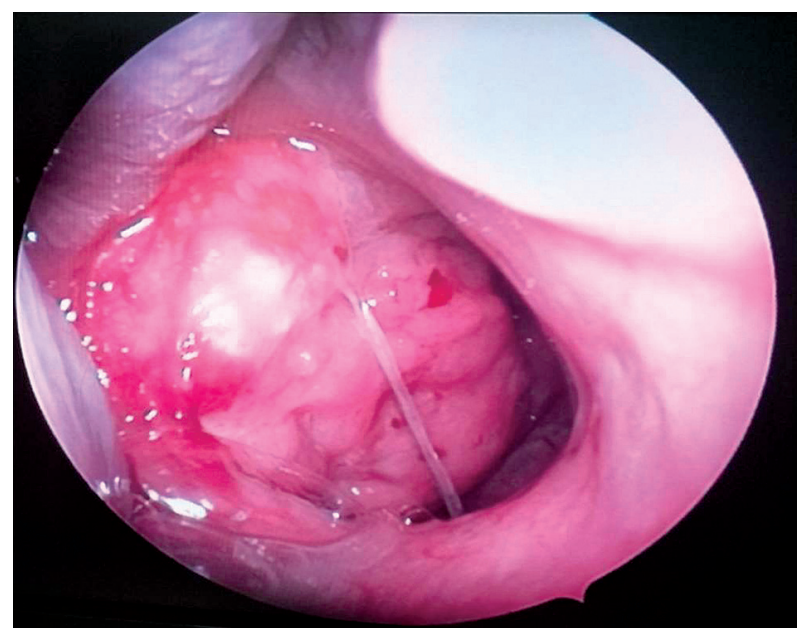

FIGURE 2. Endoscopic picture showing an irregular mass at the right side of the nasopharynx

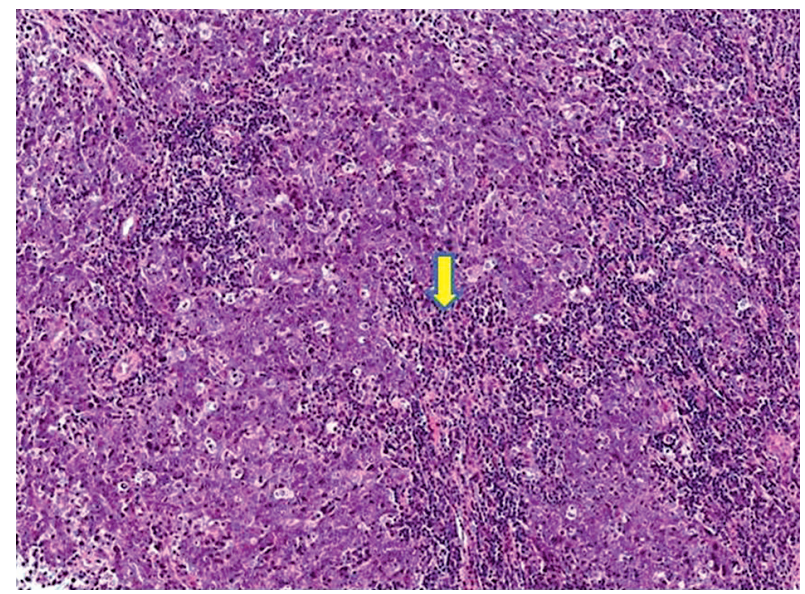

FIGURE 4. Histopathology picture of nasopharyngeal carcinoma showing invasive cords and nests of oval to cuboidal cells with large hyperchromatic and mitotically active nuclei (arrow mark)

and nests of oval to cuboidal cells with large hyperchromatic and mitotically active nuclei (Fig. 4). The diagnosis was confirmed as poorly differentiated non-keratinising squamous cell carcinoma. Chest $\mathrm{X}$-ray and abdominal ultrasound were done and showed no evidence of metastatic lesions. Epstein-Barr virus viral capsid antigen screening was negative. The patient was treated with chemotherapy and radiotherapy. The first two cycles of chemotherapy with cisplatin, bleomycin, and methotrexate were given followed by radiotherapy. The child was given 30 cycles of radiotherapy to the primary site and neck. The child achieved satisfactory control of the disease, with no evidence of recurrence in the nasopharynx one year after the diagnosis. Currently the patient is symptom free after one year of follow-up.

\section{DISCUSSION}

Nasopharyngeal carcinoma (NPC) is a common malignant tumour seen in the nasopharynx. The incidence of NPC increases with age and peaks at 50 to 59 years old 
with another, smaller peak in late childhood [3]. The incidence of paediatric NPC varies from endemic to non-endemic areas. The annual incidence of NPC is around 0.5 per million children (10-14 years of age) and 1.08 million adolescents (15 to 17 years of age) [4]. The incidence of childhood NPC is less than $1 \%$ of all paediatric malignant neoplasms [3]. NPC is pathologically classified (WHO classification) into three categories: keratinising squamous cell carcinoma (WHO type I), non-keratinising squamous cell carcinoma (WHO type II), and undifferentiated carcinoma (WHO type III). The majority of the cases are undifferentiated carcinoma (WHO type III) in advanced stage [5]. NPC is usually caused due to genetic susceptibility, environmental factors like exposure to chemical carcinogens, and infections with Epstein-Barr virus. Raised antibody titres to Epstein-Barr virus antigens are diagnostic markers [6]. The clinical presentations of paediatric NPC are nasal bleeding, nasal block, and otitis media. Due to the rich lymphatic drainage of the nasopharynx, bilateral neck node metastases are often the first clinical sign. The rarity of NPC in the paediatric age group often leads to delayed diagnosis, so childhood NPC is associated with advanced loco-regional spread and higher chance of distant metastasis [7]. In this case the diagnosis was delayed because the cervical lymphadenopathy was suspected to be of infectious cause. Unilateral serous otitis media is a more common clinical presentation in adult patients with NPC, in comparison to children. Otalgia and cervical lymphadenopathy are sugestive of infectious aetiology in the paediatric age group. In the absence of improvement with a course of antibiotic treatment, further workup is required. Persistent unilateral otological findings need flexible nasopharyngolaryngoscopic examination to rule out nasopharyngeal carcinoma. The peak incidence of NPC in children of non-endemic regions is in the age range of 15 to 19 years with a male to female ratio of $>2: 1$ [8]. Because there is no exact time-frame for initiating a malignancy work up in the paediatric age group with one-sided otological symptoms, a higher degree of suspicion may be needed in childhood. In comparison to adults, NPC in the paediatric age group has certain characteristics: undifferentiated carcinoma is the commonest histological type; in most of the cases advanced loco-regional disease is seen at first diagnosis; higher prevalence of distant metastasis; and it is closely associated with Epstein-Barr virus aetiology and higher prevalence of treatment/therapy related complications $[9,10]$.

Radiotherapy is the treatment of choice in NPC, whereas neo-adjuvant chemotherapy is given in advanced cases. Radiation of $1.8 \mathrm{~Gy} /$ day is given for five days a week, and a total dose of $40-44$ Gy is given to the primary site and neck nodes with a desirable dose of $50 \mathrm{~Gy}$. In this case, two cycles of chemotherapy were given along with radiotherapy (40 Gy). The chemotherapy included methotrexate, bleomycin, and cisplatin. Nasopharyngeal carcinomas are more radiosensitive. Our patient showed significant improvement after radiotherapy and chemotherapy and is now under follow-up. The side effects of radiotherapy to the head and neck area vary, and most often patients complain of some degree of xerostomia. A chemotherapeutic agent like cisplatin is commonly prescribed and leads to ototoxicity, typically bilateral and irreversible [11]. Loss of hearing in childhood can affect speech, as well as cognitive and social development. Future treatment needs to avoid such drugs. NPC is usually inoperable due to the complex anatomical location of the disease, and surgery of this primary neoplasm is confined to biopsy only. Radical neck dissection is considered if the primary tumour appears to be controlled or there are persistent neck nodes after chemoradiation or isolated recurrence in the neck after radiation [12]. Paediatric patients with NPC respond better than adult patients to treatment, and so the outcomes tend to be better. The five-year survival of paediatric patients under the age of 18 years is $77 \% \pm 7.3 \%$, whereas NPC-specific five-year survival is $83 \% \pm 3.9 \%[13,14]$. The risk of developing a second cancer after treatment is significantly higher in the case of paediatric NPC [7]. In children, early diagnosis of NPC before loco-regional spread will hopefully avoid the extent of radiation-associated comorbidities. The radiation dose should be reduced for patients of NPC with positive outcome to neo-adjuvant chemotherapy.

\section{CONCLUSIONS}

NPC in the paediatric age group is very rare. It presents with advanced loco-regional disease if diagnosed in late. Early administration of treatment is required for childhood NPC. The treatment of paediatric NPC is essentially multimodal and includes radiotherapy and chemotherapy. A high dose of radiation gives excellent control of NPC at the cost of toxicity with serious consequences in the paediatric age group. A lower radiation dose in paediatric NPC with the help of neo-adjuvant or concomitant chemotherapy reduces late toxicity along with local control of the tumour.

\section{DISCLOSURE}

The authors declare no conflict of interest.

\section{REFERENCES}

1. Sun LM, Li CI, Huang EY, Vaughan TL. Survival differences by race in nasopharyngeal carcinoma. Am J Epidemiol 2007; 165: 271-278.

2. Kao WC, Chen JS, Yen CJ. Advanced nasopharyngeal carcinoma in children. J Cancer Res Pract 2016; 3: 84-88.

3. Chang ET, Adami HO. The enigmatic epidemiology of nasopharyngeal carcinoma. Cancer Epidemiol Biomarkers Prev 2006; 15: 17651777.

4. Pastore G, De Salvo GL, Bisogno G, et al. Evaluating the access to pediatric cancer care centers of children and adolescents with rare 
tumors in Italy: the TREP project. Pediatr Blood Cancer 2009; 53: 152-155.

5. Lo KW, To KF, Huang DP. Focus on nasopharyngeal carcinoma. Cancer Cell 2004; 5: 423-428.

6. Chong VF, Ong CK. Nasopharyngeal carcinoma. Eur J Radiol 2008; 66: 437-447.

7. Sultan I, Casanova M, Ferrari A, et al. Differential features of nasopharyngeal carcinoma in children and adults: a SEER study. Pediatr Blood Cancer 2010; 55: 279-284.

8. Zhang L, Chen QY, Liu H, et al. Emerging treatment options for nasopharyngeal carcinoma. Drug Des Devel Ther 2013; 7: 37-52.

9. Afqir S, Ismaili N, Alaoui K, et al. Nasopharyngeal carcinoma in adolescents: a retrospective review of 42 patients. Eur Arch Otorhinolaryngol 2009; 266: 1767-1773.

10. Youssef AA, Raafat TA. Nasopharyngeal carcinoma: Imaging features of unusual cancer in children. Egypt J Radiol Nucl Med 2015; 46: 943-947.

11. Lu S, Wei J, Sun F, et al. Late Sequelae of Childhood and Adolescent Nasopharyngeal Carcinoma Survivors After Radiation Therapy. Int J Radiat Oncol Biol Phys 2019; 103: 45-51.

12. DeRenzo C, Lam C, Rodriguez-Galindo C, et al. Salvage regimens for pediatric patients with relapsed nasopharyngeal carcinoma. Pediatr Blood Cancer 2019; 66: e27469.

13. Patel PN, Penn EB. Presentation and management of nasopharyngeal carcinoma, a rare childhood malignancy. Int J Pediatr Otorhinolaryngol Extra 2016; 14: 20-22.

14. Huang Y, Zhang J, He Q, et al. Clinical outcome and prognostic analysis of young adults nasopharyngeal carcinoma patients of a nonendemic area in intensity-modulated radiotherapy era. Future Oncol 2019; 15: 381-389. 